\title{
ON THE STRONG UNICITY OF BEST CHEBYSHEV APPROXIMATION OF DIFFERENTIABLE FUNCTIONS
}

\author{
ANDRÁS KROÓ
}

\begin{abstract}
Let $X$ be a normed linear space, $U_{n}$ an $n$-dimensional Chebyshev subspace of $X$. For $f \in X$ denote by $p(f) \in U_{n}$ its best approximation in $U_{n}$. The problem of strong unicity consists in estimating how fast the nearly best approximants $g \in U_{n}$ satisfying $\|f-g\| \leqslant\|f-p(f)\|+\delta$ approach $p(f)$ as $\delta \rightarrow 0$. In the present note we study this problem in the case when $X=C^{r}[a, b]$ is the space of $r$-times continuously differentiable functions endowed with the supremum norm $(1 \leqslant r \leqslant \infty)$.
\end{abstract}

Introduction. Let $X$ be a normed linear space and let $U_{n}$ be an $n$-dimensional subspace of $X$. We say that $p \in U_{n}$ is a best approximation of $f \in X$ if $\|f-p\|=$ $\operatorname{dist}\left(f, U_{n}\right)=\inf \left\{\|f-g\|: g \in U_{n}\right\}$. (Since $U_{n}$ is finite dimensional each $f \in X$ possesses a best approximant.) Assume, in addition, that $U_{n}$ is a Chebyshev subspace of $X$, i.e. each $f \in X$ has a unique best approximation in $U_{n}$. Let us denote by $p(f) \in U_{n}$ the best approximant of $f$. A question of considerable interest is that of how fast the "nearly best approximations" $g \in U_{n}$ satisfying

$$
\|f-g\| \leqslant\|f-p(f)\|+\delta
$$

approach $p(f)$ as $\delta \rightarrow 0$. This is the so-called strong unicity problem. In the case when, for a given $f \in X$ and any $g \in U_{n}$ satisfying (1) with some $0<\delta \leqslant 1$, the relation $\|g-p(f)\| \leqslant C(f) \delta^{\gamma}$ holds, we say the degree of strong unicity at $f$ is $\gamma$ $(0<\gamma \leqslant 1, C(f)>0$ is independent of $g)$.

Consider the classical case of Chebyshev approximation, when $X=C[a, b]$ is the space of real or complex continuous functions endowed with the supremum norm $\|f\|_{C}=\max \{|f(x)|: x \in[a, b]\}$. The famous Haar-Kolmogorov theorem states that $U_{n} \subset C[a, b]$ is a Chebyshev subspace of $C[a, b]$ if and only if each nontrivial element of $U_{n}$ has at most $n-1$ distinct zeros at $[a, b]$. The finite-dimensional Chebyshev subspaces of $C[a, b]$ are usually called Haar spaces.

The strong unicity problem for $C[a, b]$ was solved by D. Newman and H. Shapiro [5]. They proved that if $U_{n}$ is a Haar space then for each $f \in C[a, b]$ the degree of strong unicity at $f$ is $\frac{1}{2}$ in the complex case and 1 in the real case. (For the $L_{p}$ spaces the problem of strong unicity was solved in [1] for $1<p<\infty$ and in [3] for $p=1$.)

Now let $X=C^{r}[a, b]=\left\{f \in C[a, b]: f^{(r)} \in C[a, b]\right\}$ be the space of $r$-times continuously differentiable functions on $[a, b]$ endowed with the same supremum norm $(1 \leqslant r \leqslant \infty)$. The characterization of Chebyshev subspaces of $C^{r}[a, b]$ was

Received by the editors January 18, 1983.

1980 Mathematics Subject Classification. Primary 41 A52. 
given in [2 and 4]. (In [2] the Chebyshev subspaces of $C^{r}[a, b]$ were studied in the real case. Later in [4] using another approach we characterized the Chebyshev subspaces of $C^{r}[a, b]$ in the complex case.) It turned out that the characteristic property of Chebyshev subspaces of $C^{r}[a, b]$ (which is actually independent of $1 \leqslant r \leqslant \infty)$ is more general than the Haar property.

The aim of this paper is to give a solution to the problem of strong unicity in $C^{r}[a, b]$.

Preliminaries. We start with a lemma which characterizes the best approximants of $f \in C[a, b]$.

LEMma [6, P. 178]. Let $U_{n}$ be an n-dimensional subspace of $C[a, b], f \in C[a, b]$. Then $p \in U_{n}$ is a best approximation of $f$ if and only if there exist points $a \leqslant x_{1}<x_{2}$ $<\cdots<x_{m} \leqslant b$, where $1 \leqslant m \leqslant n+1$ in the real case and $1 \leqslant m \leqslant 2 n+1$ in the complex case, and numbers $a_{i} \neq 0,1 \leqslant i \leqslant m$, such that

$$
f\left(x_{i}\right)-p\left(x_{i}\right)=\left(\bar{a}_{i} /\left|a_{i}\right|\right)\|f-p\|_{C} \quad(1 \leqslant i \leqslant m)
$$

and

$$
\sum_{i=1}^{m} a_{i} g\left(x_{i}\right)=0
$$

for any $g \in U_{n}$.

Definition 1. The set of points $a \leqslant x_{1}<\cdots<x_{m} \leqslant b$, where $1 \leqslant m \leqslant n+1$ in the real case and $1 \leqslant m \leqslant 2 n+1$ in the complex case, is called an extremal set of the $n$-dimensional subspace $U_{n} \subset C[a, b]$ if there exist numbers $a_{i} \neq 0,1 \leqslant i \leqslant m$ (real or complex, respectively) such that (3) holds for every $g \in U_{n}$. The numbers $\left\{a_{i}\right\}_{i=1}^{m}$ are called coefficients of the extremal set $\left\{x_{i}\right\}_{i=1}^{m}$. (Note that the coefficients of an extremal set are, in general, defined nonuniquely.)

It can be shown that a necessary and sufficient condition for $U_{n}$ to be a Haar space is that no nontrivial element of $U_{n}$ can vanish on an extremal set of $U_{n}$. This statement can be considered as another definition of Haar spaces.

Definition 2. Let $U_{n}$ be an $n$-dimensional subspace of $C^{1}[a, b]$. Then $U_{n}$ is called a semi-Haar space if and only if there does not exist an extremal set $\left\{x_{i}\right\}_{i=1}^{m}$ of $U_{n}$ with coefficients $\left\{a_{i}\right\}_{i=1}^{m}$ and $g \in U_{n} \backslash\{0\}$ such that $g\left(x_{i}\right)=0$ for any $1 \leqslant i \leqslant m$ and $\operatorname{Re} a_{i} g^{\prime}\left(x_{i}\right)=0$ if $x_{i} \in(a, b)(1 \leqslant i \leqslant m)$. (Remark: In the real case the relation $\operatorname{Re} a_{i} g^{\prime}\left(x_{i}\right)=0$ is equivalent to $g^{\prime}\left(x_{i}\right)=0$.)

It turned out (see [4]) that $U_{n} \subset C^{r}[a, b]$ is a Chebyshev subspace of $C^{r}[a, b]$ if and only if $U_{n}$ is a semi-Haar space $(1 \leqslant r \leqslant \infty)$. Therefore it is natural to consider the problem of strong unicity of best Chebyshev approximation of differentiable functions with respect to semi-Haar spaces. (It is shown in [4] that the family of semi-Haar spaces is essentially wider than that of Haar spaces, in particular there are different spaces of real and complex lacunary polynomials, i.e. polynomials with gaps, which do not satisfy the Haar property but are nevertheless semi-Haar spaces.) 
New results. In this section we shall present some strong unicity type results for semi-Haar spaces. Let us give several additional notations. For $f \in C[a, b]$ we denote by

$$
\omega(f, h)=\sup \left\{\left|f\left(x_{1}\right)-f\left(x_{2}\right)\right|: x_{1}, x_{2} \in[a, b],\left|x_{1}-x_{2}\right| \leqslant h\right\}
$$

the modulus of continuity of $f(0<h \leqslant b-a)$. Furthermore, set

$$
\text { Lip } \alpha=\left\{f \in C[a, b]: \sup h^{-\alpha} \omega(f, h)<\infty\right\}, \quad 0<\alpha \leqslant 1 .
$$

Let $\varphi_{1}, \ldots, \varphi_{n}$ be a basis in $U_{n} \subset C^{1}[a, b]$ and consider the modulus $\omega^{*}(h)=$ $\sum_{i=1}^{\prime \prime} \omega\left(\varphi_{i}^{\prime}, h\right)$. Then for any $p \in U_{n}$,

$$
\omega\left(p^{\prime}, h\right) \leqslant K_{0}\|p\|_{C} \omega^{*}(h),
$$

where $K_{0}>0$ is independent of $p$. If $U_{n}$ is a semi-Haar space then $p(f) \in U_{n}$ denotes the unique best approximation of $f \in C^{1}[a, b]$.

ThIOREM 1. Let $U_{n}$ be a semi-Haar space. Then for any given $f \in C^{1}[a, b]$ and every $g \in U_{n}$ such that

$$
\|f-g\|_{C} \leqslant\|f-p(f)\|_{C}+\delta\left(\omega\left(f^{\prime}, \delta\right)+\omega^{*}(\delta)+\delta\right)
$$

with some $0<\delta \leqslant b-a$, we have

$$
\|p(f)-g\|_{c} \leqslant K_{1}\left(\omega\left(f^{\prime}, \delta\right)+\omega^{*}(\delta)+\delta\right),
$$

where the constant $K_{1}>0$ is independent of $g$ and $\delta$.

In particular, if $f^{\prime}, \varphi_{1}^{\prime}, \ldots, \varphi_{n}^{\prime} \in \operatorname{Lip} \alpha$, where $\varphi_{1}, \ldots, \varphi_{n}$ are the basis functions of $U_{n}$, then the degree of strong unicity at $f$ is $\alpha /(1+\alpha)$. Thus for semi-Haar spaces the degree of strong unicity depends, in general, on the constructive properties of the functions considered. On the other hand, Theorem 1 implies that in $C^{2}[a, b]$ the degree of strong unicity (with respect to semi-Haar spaces) is $\frac{1}{2}$ for each $f \in C^{2}[a, b]$.

Corollary. Let $U_{n} \subset C^{2}[a, b]$ be a semi-Haar space. Then for each $f \in C^{2}[a, b]$ the degree of strong unicity at $f$ is equal to $\frac{1}{2}$.

The above corollary shows the surprising fact that in $C^{2}[a, b]$ the Newman-Shapiro theorem (complex version) remains true under the weaker semi-Haar assumption.

On the other hand, as the following theorem shows in $C^{1}[a, b]$, the degree of strong unicity for non-Haar spaces is, in general, less than $\frac{1}{2}$.

ThEOREM 2. Assume $U_{n} \subset C^{1}[a, b]$ does not satisfy the Haar property, i.e. some $g \in U_{n} \backslash\{0\}$ has $n$ distinct zeros at $[a, b]$. Then for any $0<\alpha \leqslant 1$ there exists $a$ function $f_{\alpha} \in C^{1}[a, b]$ such that $f_{\alpha}^{\prime} \in \operatorname{Lip} \alpha, 0$ is a best approximant of $f_{\alpha}$ in $U_{n}$, and

$$
\left\|f_{\alpha}-\delta^{\alpha /(\alpha+1)} g\right\|_{C} \leqslant\left\|f_{\alpha}\right\|_{C}+K_{2} \delta \quad(0<\delta \leqslant 1)
$$

where $K_{2} \geqslant 0$ is independent of $\delta$. Moreover, if $\alpha=1$ then $f_{1} \in C^{\infty}[a, b]$.

Thus the degree of strong unicity given by Theorem 1 is sharp, in general, and cannot be improved even for "very smooth" functions or real functions. 
Proof of Theorem 1. Let $f \in C^{1}[a, b] \backslash U_{n}$ (the case $f \in U_{n}$ is trivial) and assume that $g \in U_{n}, g \neq p(f)$ satisfies (5) with some $0<\delta \leqslant b-a$. Set $f_{0}=f-p(f)$, $g_{0}=g-p(f)\left(g_{0} \in U_{n} \backslash\{0\}\right)$. Then 0 is the best approximation of $f_{0}$ and, by (5),

$$
\left\|f_{0}-g_{0}\right\|_{C} \leqslant\left\|f_{0}\right\|_{C}+\delta A(\delta)
$$

where $A(h)=\omega\left(f^{\prime}, h\right)+\omega^{*}(h)+h(0<h \leqslant b-a)$. Furthermore, we have, by (4),

$$
\begin{aligned}
\omega\left(f_{0}^{\prime}, h\right) & \leqslant \omega\left(f^{\prime}, h\right)+\omega\left(p^{\prime}(f), h\right) \leqslant \omega\left(f^{\prime}, h\right)+K_{0}\|p(f)\|_{C} \omega^{*}(h) \\
& \leqslant c_{1} A(h) .
\end{aligned}
$$

(Here and in what follows the positive constants depending only on $f, a, b$ and $U_{n}$ are denoted by $c_{i}, i=1,2, \ldots$ )

Since 0 is the best approximant of $f_{0}$ in $U_{n}$, it follows by the Lemma that there exists an extremal set $\left\{x_{i}\right\}_{i=1}^{m}$ of $U_{n}$ with coefficients $\left\{a_{i}\right\}_{i=1}^{m}$ such that

$$
f_{0}\left(x_{i}\right)=\left(\bar{a}_{i} /\left|a_{i}\right|\right)\left\|f_{0}\right\|_{c} \quad(1 \leqslant i \leqslant m) .
$$

Hence, by (8),

$$
\begin{aligned}
& \left|f_{0}\left(x_{i}\right)-g_{0}\left(x_{i}\right)\right|^{2}=\left|\left\|f_{0}\right\|_{C}-\left(a_{i} /\left|a_{i}\right|\right) g_{0}\left(x_{i}\right)\right|^{2} \\
& =\left\|f_{0}\right\|_{C}^{2}-\left(2\left\|f_{0}\right\|_{C} /\left|a_{i}\right|\right) \operatorname{Re} a_{i} g_{0}\left(x_{i}\right)+\left|g_{0}\left(x_{i}\right)\right|^{2} \\
& \leqslant\left\|f_{0}-g_{0}\right\|_{C}^{2} \leqslant\left(\left\|f_{0}\right\|_{C}+\delta A(\delta)\right)^{2} \leqslant\left\|f_{0}\right\|_{c}^{2}+c_{2} \delta A(\delta) \quad(1 \leqslant i \leqslant m) .
\end{aligned}
$$

This obviously implies that

Moreover, by definition of extremal sets, $\sum_{i=1}^{m} a_{i} g_{0}\left(x_{i}\right)=0$. Hence, by (12), for any $1 \leqslant j \leqslant m$,

$$
\operatorname{Re} a_{j} g_{0}\left(x_{j}\right)=-\sum_{\substack{i=1 \\ i \neq j}}^{m} \operatorname{Re} a_{i} g_{0}\left(x_{i}\right) \leqslant c_{4} \delta A(\delta) \text {. }
$$

Thus we finally obtain that

$$
\left|\operatorname{Re} a_{i} g_{0}\left(x_{i}\right)\right| \leqslant c_{5} \delta A(\delta) \quad(1 \leqslant i \leqslant m) .
$$

Furthermore, using this inequality in (11) we have

$$
\left|g_{0}\left(x_{i}\right)\right| \leqslant \sqrt{c_{6} \delta A(\delta)} \leqslant \sqrt{c_{6}} A(\delta)=c_{7} A(\delta) \quad(1 \leqslant i \leqslant m) .
$$

Consider an arbitrary point $x_{i}$ of the extremal set belonging to $(a, b)$. Set

$$
f_{1}(x)=\left(1 /\left|a_{i}\right|\right) \operatorname{Re} a_{i} f_{0}(x), \quad g_{1}(x)=\left(1 /\left|a_{i}\right|\right) \operatorname{Re} a_{i} g_{0}(x) .
$$

Then the real functions $f_{1}, g_{1} \in C^{1}[a, b]$ have the following properties:

$$
\begin{gathered}
f_{1}\left(x_{i}\right)=\left\|f_{0}\right\|_{C}=\left\|f_{1}\right\|_{C}, \quad f_{1}^{\prime}\left(x_{i}\right)=0 ; \\
\left\|f_{1}-g_{1}\right\|_{C} \leqslant\left\|f_{0}-g_{0}\right\|_{C} \leqslant\left\|f_{0}\right\|_{C}+\delta A(\delta)=\left\|f_{1}\right\|_{C}+\delta A(\delta) ; \\
\left|g_{1}\left(x_{i}\right)\right| \leqslant c_{8} \delta A(\delta),
\end{gathered}
$$

where (15), (16) and (17) follow immediately from (10), (8) and (13), respectively. 
Using equations (16), (15) and (9) we obtain that for any real $h$ such that $|h| \leqslant$ $\min \left\{x_{i}-a, b-x_{i}\right\}$,

$$
\begin{aligned}
g_{1}\left(x_{i}+h\right) & \geqslant f_{1}\left(x_{i}+h\right)-\left\|f_{1}-g_{1}\right\|_{C} \geqslant f_{1}\left(x_{i}+h\right)-\left\|f_{1}\right\|_{C}-\delta A(\delta) \\
& =-\delta A(\delta)+f_{1}\left(x_{i}+h\right)-f_{1}\left(x_{i}\right) \geqslant-\delta A(\delta)-|h| \omega\left(f_{1}^{\prime},|h|\right) \\
& \geqslant-\delta A(\delta)-|h| \omega\left(f_{0}^{\prime},|h|\right) \geqslant-\delta A(\delta)-c_{1}|h| A(|h|) .
\end{aligned}
$$

Furthermore for some $\xi \in[a, b]$ such that $\left|\xi-x_{i}\right| \leqslant|h|$, we have

$$
g_{1}^{\prime}\left(x_{i}\right) h=g_{1}\left(x_{i}+h\right)-g_{1}\left(x_{i}\right)+h\left(g_{1}^{\prime}\left(x_{i}\right)-g_{1}^{\prime}(\xi)\right) \text {. }
$$

This, together with (18), (17), (4) and (8), yields

$$
\begin{aligned}
g_{1}^{\prime}\left(x_{i}\right) h & \geqslant g_{1}\left(x_{i}+h\right)-g_{1}\left(x_{i}\right)-|h| \omega\left(g_{1}^{\prime},|h|\right) \\
& \geqslant-\delta A(\delta)-c_{1}|h| A(|h|)-c_{8} \delta A(\delta)-|h| \omega\left(g_{0}^{\prime},|h|\right) \\
& \geqslant-c_{9} \delta A(\delta)-c_{1}|h| A(|h|)-K_{0}\left\|g_{0}\right\|_{C} \omega^{*}(|h|)|h| \\
& \geqslant-c_{9} \delta A(\delta)-c_{1}|h| A(|h|)-K_{0}\left(2\left\|f_{0}\right\|_{C}+(b-a) A(b-a)\right)|h| \omega^{*}(|h|) \\
& \geqslant-c_{9} \delta A(\delta)-c_{10}|h| A(|h|) \geqslant-c_{11}(\delta A(\delta)+|h| A(|h|)) .
\end{aligned}
$$

Since the above inequality holds for any $h$ such that $|h|$ is small enough, we may set $h= \pm c_{12} \delta$ (with appropriate $0<c_{12}<1$ ) which gives us two estimations:

$$
\begin{aligned}
& g_{1}^{\prime}\left(x_{i}\right) \geqslant-c_{11}\left(\delta A(\delta)+c_{12} \delta A\left(c_{12} \delta\right)\right) / c_{12} \delta \geqslant-c_{13} A(\delta), \\
& g_{1}^{\prime}\left(x_{i}\right) \leqslant c_{11}\left(\delta A(\delta)+c_{12} \delta A\left(c_{12} \delta\right)\right) / c_{12} \delta \leqslant c_{13} A(\delta) .
\end{aligned}
$$

Hence $\left|g_{1}^{\prime}\left(x_{i}\right)\right| \leqslant c_{13} A(\delta)$, i.e.

$$
\left|\operatorname{Re} a_{i} g_{0}^{\prime}\left(x_{i}\right)\right| \leqslant c_{14} A(\delta)
$$

for those $1 \leqslant i \leqslant m$ for which $x_{i}$ belong to $(a, b)$.

Let us consider, on $U_{n}$, the functional

$$
F(p)=\max _{1 \leqslant i \leqslant m}\left|p\left(x_{i}\right)\right|+\max _{\substack{1 \leqslant i \leqslant m \\ x_{i} \in(a, h)}}\left|\operatorname{Re} a_{i} p^{\prime}\left(x_{i}\right)\right| \quad\left(p \in U_{n}\right) .
$$

Since $\left\{x_{i}\right\}_{i=1}^{m}$ is an extremal set of $U_{n}$ with coefficients $\left\{a_{i}\right\}_{i=1}^{m}$, and $U_{n}$ is a semi-Haar space, it follows by definition of semi-Haar spaces that $F(p)>0$ for every $p \in U_{n} \backslash\{0\}$. Moreover, it can be easily seen that $F$ is continuous on $U_{n}$ (endowed with the supremum norm). Hence by compactness of the unit ball in $U_{n}$,

$$
\|p\|_{C} \leqslant c_{15} F(p)
$$

for each $p \in U_{n}$. Furthermore, by (14) and (19),

$$
F\left(g_{0}\right) \leqslant\left(c_{7}+c_{14}\right) A(\delta) .
$$

Combining the above estimation with $(20)$ we finally obtain

$$
\|g-p(f)\|_{C}=\left\|g_{0}\right\|_{C} \leqslant c_{15} F\left(g_{0}\right) \leqslant c_{15}\left(c_{7}+c_{14}\right) A(\delta) .
$$

The proof of Theorem 1 is completed.

REMARK. Actually the Chebyshev property of semi-Haar spaces was not used in the proof of Theorem 1. Therefore Theorem 1 implies, in particular, that semi-Haar spaces are Chebyshev subspaces of $C^{1}[a, b]$. 
Proof of Theorem 2. Let $x_{1}, \ldots, x_{n} \in[a, b]$ be distinct zeros of $g \in U_{n} \backslash\{0\}$. Consider the linear functionals $L_{i} \in U_{n}^{*}$ given by $L_{i}(p)=p\left(x_{i}\right)\left(p \in U_{n}\right), 1 \leqslant i \leqslant n$. Evidently, $L_{i}, 1 \leqslant i \leqslant n$, are linearly dependent on $U_{n}$ (otherwise they would span $U_{n}^{*}$, which is impossible since $\left.g\left(x_{i}\right)=0,1 \leqslant i \leqslant n\right)$. This immediately implies $\left\{x_{i}\right\}_{i=1}^{n}$, or a proper subset of it, is an extremal set of $U_{n}$. Hence, without loss of generality, we may assume $\left\{x_{i}\right\}_{i=1}^{m}$ is an extremal set of $U_{n}(1 \leqslant m \leqslant n)$ with corresponding nonzero coefficients $\left\{a_{i}\right\}_{i=1}^{m}$, and $g\left(x_{i}\right)=0,1 \leqslant i \leqslant m$.

There exists a function $\tilde{F} \in C^{x}[a, b]$ such that $\tilde{F}\left(x_{i}\right)=\bar{a}_{i} /\left|a_{i}\right|(1 \leqslant i \leqslant m)$ and $\|\tilde{F}\|_{c}=1$.

Set

$$
f_{\alpha}(x)=\tilde{F}(x)\left(1-\gamma \prod_{i=1}^{m}\left|x-x_{i}\right|^{1+\alpha}\right), \quad 0<\alpha \leqslant 1,
$$

where $\gamma>0$ is chosen so that $\gamma \prod_{i=1}^{m}\left|x-x_{i}\right|^{1+\alpha} \leqslant 1$, i.e. $\left\|f_{\alpha}\right\|_{C}=1$. Since $f_{\alpha}\left(x_{i}\right)=$ $\tilde{F}\left(x_{i}\right)=\bar{a}_{i} /\left|a_{i}\right|(1 \leqslant i \leqslant m)$ it follows by the Lemma that 0 is a best approximant of $f_{\alpha}$. Moreover, it can be easily seen that $f_{\alpha}^{\prime} \in \operatorname{Lip} \alpha(0<\alpha<1)$ and $f_{1} \in C^{\infty}[a, b]$.

Let us estimate the quantity $\left\|f_{\alpha}-\delta^{\alpha /(\alpha+1)} g\right\|_{C}$. Since $g\left(x_{i}\right)=0,1 \leqslant i \leqslant m$, we can obtain, for any $x \in[a, b]$,

$$
|g(x)| \leqslant\left(\left\|\operatorname{Re} g^{\prime}\right\|_{C}+\left\|\operatorname{Im} g^{\prime}\right\|_{C}\right) \min _{1 \leqslant i \leqslant m}\left|x-x_{i}\right| .
$$

Furthermore, it can be easily shown that

$$
\prod_{i=1}^{m}\left|x-x_{i}\right|^{1+\alpha} \geqslant c_{16}\left(\min _{1 \leqslant i \leqslant m}\left|x-x_{i}\right|\right)^{1+\alpha} \quad(x \in[a, b]),
$$

where $c_{16}>0$ does not depend on $x$. Hence, by (21) and (22), for any $x \in[a, b]$,

$$
\begin{aligned}
& \left|f_{\alpha}(x)-\delta^{\alpha /(\alpha+1)} g(x)\right| \leqslant\left|f_{\alpha}(x)\right|+\delta^{\alpha /(\alpha+1)}|g(x)| \\
& \leqslant 1-\gamma \prod_{i=1}^{m}\left|x-x_{i}\right|^{1+\alpha}+\delta^{\alpha /(\alpha+1)}\left(\left\|\operatorname{Re} g^{\prime}\right\|_{C}+\left\|\operatorname{Im} g^{\prime}\right\|_{C}\right) \min _{1 \leqslant i \leqslant m}\left|x-x_{i}\right| \\
& \quad \leqslant 1-c_{17}\left(\min _{1 \leqslant i \leqslant m}\left|x-x_{i}\right|\right)^{1+\alpha}+c_{18} \delta^{\alpha /(\alpha+1)} \min _{1 \leqslant i \leqslant m}\left|x-x_{i}\right| \\
& \quad \leqslant \max _{\xi>0}\left(1-c_{17} \xi^{1+\alpha}+c_{18} \delta^{\alpha /(\alpha+1)} \xi\right)=1+c_{19} \delta .
\end{aligned}
$$

Thus $\left\|f_{\alpha}-\delta^{\alpha /(\alpha+1)} g\right\|_{C} \leqslant 1+c_{19} \delta$ for any $\delta>0$.

The proof of Theorem 2 is completed.

Note that the strong unicity type result given by Theorem 1 can be applied as usual to the study of modulus of continuity of the operator of best approximation and discretization errors.

\section{REFERENCES}

1. B. O. Björnestàl, Local Lipschitz continuity of the metric projection operator. Banach Center Publications, 4. Approximation Theory. PWN, Warsaw, 1979, pp. 43-53.

2. A. L. Garkavi, Dimensionality of polyhedra of best approximation for differentiable functions. Izv. Akad. Nauk SSSR Ser. Mat. 23 (1959), 93-114. (Russian) 
3. A. Kroó, On strong unicity of $L_{1}$-approximation, Proc. Amer. Math. Soc. 83 (1981), 725-729.

4. On the unicity of best Chebyshev approximation of differentiable functions, Acta Sci. Math. Szeged (to appear).

5. D. Newman and H. Shapiro, Some theorems on Čebyšev approximation, Duke Math. J. 30 (1963), 673-681.

6. I. Singer, Best approximation in normed linear spaces by elements of linear subspaces, Springer-Verlag, Berlin and New York, 1970.

Mathematical Institute of the Hungarian Academy of Sciences, Budapest H-1395 Pf. 428, HUNGARY 